\title{
Rolling and Adhesion of Human Tumor Cells on Vascular Endothelium under Physiological Flow Conditions
}

Raffaella Giavazzi, Marco Foppolo, Romina Dossi, and Andrea Remuzzi

Mario Negri Institute for Pharmacological Research, 24100 Bergamo, Italy

\begin{abstract}
We investigated the interaction of different human tumor types with resting and IL-1-activated human umbilical vein endothelial cells under laminar flow conditions using a parallel plate flow chamber. Three tumor cell lines ( the HT-29M colon carcinoma, the OVCAR-3 ovarian carcinoma, and the T-47D breast carcinoma) showed limited adhesion to unstimulated endothelial cells at any of the shear stress levels tested, while rolling and massive adhesion of tumor cells were observed on IL-1-activated endothelial cells. Three other tumor cell lines (the A375M and A2058 melanomas and the MG-63 osteosarcoma) did not adhere on resting endothelial cells at high shear stress ( $>1.5 \mathrm{dyn} / \mathrm{cm}^{2}$ ) and started to adhere with decreasing shear stress; the number of adherent cells increased steeply on IL-1activated endothelial cells, but no cell rolling was observed even at the highest shear stress. These mechanisms of tumor cell interaction with endothelial cells were analyzed in detail using the HT-29M colon carcinoma and the A375M melanoma. Incubation of activated endothelial cells with a monoclonal antibody against E-selectin inhibited rolling and adhesion of HT29M, but had no effect on the adhesion of A375M cells; monoclonal antibody against vascular cell adhesion molecule-1 reduced the adhesion of $\mathrm{A375M}$ cells and had no effect on HT-29M. The selective interaction of these two molecules with tumor cells was confirmed by measuring the adhesion of tumor cells on immobilized soluble proteins. On E-selectin-coated surfaces, HT-29M cells rolled during perfusion experiments without subsequent adhesion, while A375M cells did not adhere. On vascular cell adhesion molecule-1-coated surfaces, HT-29M cells neither adhered nor rolled, while A375M cells adhered massively without rolling. Under flow conditions, therefore, cells from different tumor types interact with the endothelial surface by different mechanisms, depending on adhesion molecules expressed on the tumor and endothelial cell surface. (J. Clin. Invest. 1993. 92:3038-3044.) Key words: cell adhesion • cell rolling • endothelial cells • tumor cells • shear stress
\end{abstract}

Address correspondence to Dr. Andrea Remuzzi, Biomedical Engineering Laboratory, Mario Negri Institute for Pharmacological Research, Via Gavazzeni, 11, 24125 Bergamo, Italy.

Received for publication 28 May 1993 and in revised form 13 September 1993.

J. Clin. Invest.

(c) The American Society for Clinical Investigation, Inc.

0021-9738/93/12/3038/07 \$2.00

Volume 92, December 1993, 3038-3044

\section{Introduction}

Blood-borne tumor cells tend to metastasize through the microcirculation. Their initial arrest and attachment to vascular endothelium precedes their extravasation from the blood stream and is a crucial step in the metastatic cascade $(1,2)$. Tumor cell extravasation is equivalent, in many respects, to the entry of normal circulating cells into inflammatory tissue (3, 4). As documented by intravital microscopy, during inflammation, leukocytes begin to interact with the endothelium by first rolling along the vascular wall, then adhering to endothelial cells (EC) ${ }^{1}(5-7)$. Firmly adherent leukocytes then transmigrate through the endothelium. This recruitment of circulating leukocytes into inflammatory sites is regulated by specific recognition of EC $(8,9)$.

To study the role of these mechanisms, in vitro perfusion systems have been used to simulate the physiological conditions of blood motion in postcapillary venules, the site of major leukocyte infiltration during inflammation, in a dynamic flow environment. Thus, leukocyte rolling and adherence on cultured EC has been reproduced in vitro and the roles of adhesion molecules that mediate these interactions have been identified (10-13). The initial rolling of leukocytes along the vascular wall is mediated by selectins and the firm adhesion by integrins.

So far, tumor cell adhesion to cultured EC has been studied almost exclusively in static conditions and different adhesion structures on the tumor cell surface have been proposed as mediators of tumor-EC interactions $(1,14,15)$. Adhesion of tumor cells is significantly augmented on EC activated by cytokines (16-18). This results from the induction, or enhanced expression, of adhesion molecules on EC, as described for the adhesion of leukocytes on EC (19). Two of these molecules, E-selectin and vascular cell adhesion molecule-1 (VCAM-1), appear to play a pivotal role in the tumor-EC interaction, though tumor types of different histological origin use different adhesion mechanisms. For example, selectin-carbohydrate interactions support the adhesion of colon-related carcinomas (20-23), while VCAM-1-integrin interactions mediate the adhesion of melanomas $(20,24)$. Studies of tumor cell-EC interactions under static conditions, however, cannot document the dynamic characteristics of this interaction and the effects of physical forces (such as shear stress) in the microcirculation.

The aim of this study was to investigate the interaction of human tumor cells with vascular endothelium under dynamic flow conditions. The adhesion of tumor cells to human umbili-

1. Abbreviations used in this paper: EC, endothelial cells; HUVEC, human umbilical vein EC; VCAM-1, vascular cell adhesion molecule1. 
cal vein endothelial cell (HUVEC) cultures was studied using a parallel plate flow chamber recently used to investigate the leukocyte-endothelial interaction in vitro in well-defined laminar flow conditions $(10,12)$. The adhesion of six human tumor lines of different histological origin was investigated at different shear stresses. The specific adhesion mechanisms involved in this process were evaluated using two representative tumor types, the colon carcinoma HT-29M and the melanoma A375M, already described as adhering on cytokine-activated EC preferentially through selectin- or integrin-mediated pathways $(16,22,25)$. We also investigated the selective interaction of tumor cells with E-selectin and VCAM-1 molecules immobilized on artificial surfaces using the same flow chamber.

\section{Methods}

Cell cultures. The human tumor cell lines used were the HT-29M colon carcinoma (26), the OVCAR-3 ovarian carcinoma (27), the T-47D breast carcinoma (28), the A375M and A2058 melanomas $(29,30)$, and the MG-63 osteosarcoma (25). Tumor cells were cultured as described in the respective references. For adhesion assays, tumor cells were harvested by brief exposure to $0.25 \%$ trypsin and $0.02 \%$ EDTA, washed twice, and resuspended at a concentration of $10^{6}$ cells $/ \mathrm{ml}$ in Eagle's minimal essential medium containing $0.1 \%$ bovine serum albumin, referred to as test medium. HUVEC were isolated from the human umbilical vein and grown in culture as previously described (16, 31). Cells were grown in M199 medium supplemented with $10 \%$ fetal calf serum and $10 \%$ human serum. HUVEC purity was assessed by indirect immunofluorescence microscopy using rabbit anti-human Factor VIII antigen. HUVEC at passage 3-4, plated on $40 \times 22-\mathrm{mm}$ plastic coverslips ( Thermanox; Nunc, Naperville, IL) coated with gelatine, were used for adhesion assays $2 \mathrm{~d}$ after reaching confluence. All culture reagents were purchased from Gibco-Europe (Paisley, Scotland).

Reagents and antibodies. Human recombinant IL-1 (Escherichia coli $117-269$, specific activity $=10^{7} \mathrm{U} / \mathrm{mg}$ ) was kindly provided by $\mathrm{Dr}$. D. Boraschi (Sclavo, Siena, Italy). Monoclonal antibodies to VCAM-1 (clone 4B2) and to E-selectin (clone 13D5) and recombinant soluble proteins, VCAM-1 and E-selectin, were obtained through the courtesy of Dr. A. Gearing (British Biotechnology Ltd., Oxford, England). The antibodies were isotype (IgG1)-matched and were purified from hybridoma supernatants using protein A affinity chromatography (32). The soluble E-selectin and VCAM-1 were produced by deletion of the transmembrane and cytoplasmatic domains of full-length protein. The proteins were expressed from the pGW1HG vector in Chinese hamster ovary cells and purified on mAb affinity columns, as described (33). The purified protein was detected by a specific ELISA (British Biotechnology Products) and shown to be biologically active in cell binding assays (32).

Substrate preparation. HUVEC monolayers were activated by 4 -h incubation at $37^{\circ} \mathrm{C}$ with IL-1 $(20 \mathrm{U} / \mathrm{ml})$ in M199 medium with $10 \%$ fetal calf serum. Untreated and IL-1-treated HUVEC monolayers were washed twice with test medium after incubation and before use for adhesion experiments. Antibodies directed to adhesion proteins were added directly to stimulated or untreated HUVEC monolayers ( 1:50 final concentration), and cell monolayers were used for adhesion experiments after $30 \mathrm{~min}$ incubation at $37^{\circ} \mathrm{C}$. Adhesion surface (Thermanox coverslips) was coated with soluble proteins by adding $100 \mu \mathrm{l}$ of protein $(5 \mu \mathrm{g} / \mathrm{ml})$ on a marked area of the coverslip and incubating overnight at $4^{\circ} \mathrm{C}$. After two washes in phosphate buffer saline, coated surfaces were incubated for $1 \mathrm{~h}$ with phosphate buffer saline containing $1 \%$ of bovine serum albumin and then used for the adhesion assay. Control coverslips were coated with adhesion buffer only, following the same procedure.

Experimental apparatus. Adhesion experiments used a parallel plate laminar flow chamber similar to that described in detail by Lawrence et al. $(10,12)$. Briefly, one side of the chamber consists of a coverslip with cultured HUVEC, the other side is a flat surface ma- chined from polymethylmethacrylate. The two surfaces are separated by a $250-\mu \mathrm{m}$ thick silicon rubber gasket, leaving a rectangular adhesion surface of $30 \times 13 \mathrm{~mm}$. An inlet and an outlet channel are machined in the chamber to distribute the fluid uniformly along the entrance side of the adhesion surface.

After assembling with the HUVEC monolayer, the chamber is placed on the stage of an inverted phase-contrast microscope with a thermostated hood to maintain the temperature at $37^{\circ} \mathrm{C}$. The microscope is connected with a video recording system (Panasonic, Osaka, Japan). Test medium is pumped from a test tube through the chamber at controlled flow rates using a syringe pump. The wall shear stress on the HUVEC surface as a function of flow rate was calculated using Poiseuille's equation, as described previously for this flow chamber (12), and using the cell suspension viscosity measured at $37^{\circ} \mathrm{C}(0.8 \mathrm{cP})$.

Adhesion assay. After loading the flow chamber with a coverslip, fresh medium was initially perfused at $3.0 \mathrm{dyn} / \mathrm{cm}^{2}$ for $5 \mathrm{~min}$ for equilibration. Tumor cell suspension $\left(10^{6}\right.$ cells $\left./ \mathrm{ml}\right)$ was then perfused through the chamber at $3.0 \mathrm{dyn} / \mathrm{cm}^{2}$. To measure cell adhesion at different wall shear stresses (from 3.0 to $0.3 \mathrm{dyn} / \mathrm{cm}^{2}$ ), experiments were performed using step-down changes in flow rate, according to the protocol described in Fig. 1. The number of tumor cells firmly attached on the adhesion surface was measured at 1-min intervals during perfusion. After $12 \mathrm{~min}$ of perfusion, the flow rate of the cell suspension was raised to increase wall shear stress from 0.3 to $3.0 \mathrm{dyn} / \mathrm{cm}^{2}$ to measure the number of cells rolling on the surface and their mean velocity. At this high flow rate, tumor cells rolling on the adhesion surface are easily distinguishable from cells freely flowing in the suspension that move much faster.

Image acquisition and analysis. All adhesion experiments were video taped for subsequent quantification of adherent and rolling cells. Images were digitized from the video tape recorder using a personal computer (Apple Macintosh IIfx; Apple Computer Corp., Cupertino, CA) and processed using general purpose image analysis software (Image, v. 1.43; National Institutes of Health, Bethesda, MD). Firmly attached cells were identified and counted using an averaging technique. Briefly, an image was obtained by averaging screen pixels in four images sequentially digitized within a 4-s interval (multiframe acquisition). The result of this procedure is that cells not firmly attached to the surface, such as rolling cells and cells flowing in the suspension, are not visible on the image, and only cells remaining in the same position throughout the acquisition interval are shown. The difference between two images acquired using the single- and multiframe techniques is reported in Fig. 2. The single-frame image $(A)$ shows both adherent and not firmly adherent cells, while in the multiframe image $(B)$, only firmly attached cells are shown.

We used this technique to assess the number of cells rolling on the adhesion surface. Two images were digitized at the same perfusion time, one using single-frame and the other multiframe acquisition. We then counted rolling cells as the number of cells appearing in the single-

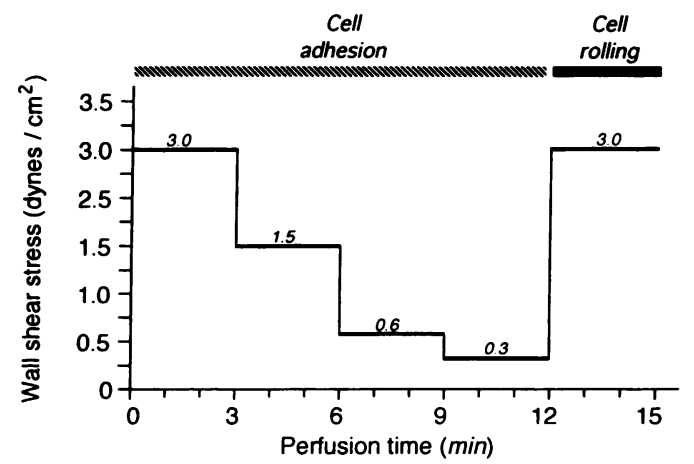

Figure 1. Wall shear stress levels during perfusion experiments. The number of cells firmly adhering to the surface was measured at 1-min intervals from 0 to $12 \mathrm{~min}$. The number of rolling cells and their mean velocity were estimated between 12 and 15 min of perfusion at 3.0 dynes $/ \mathrm{cm}^{2}$. 

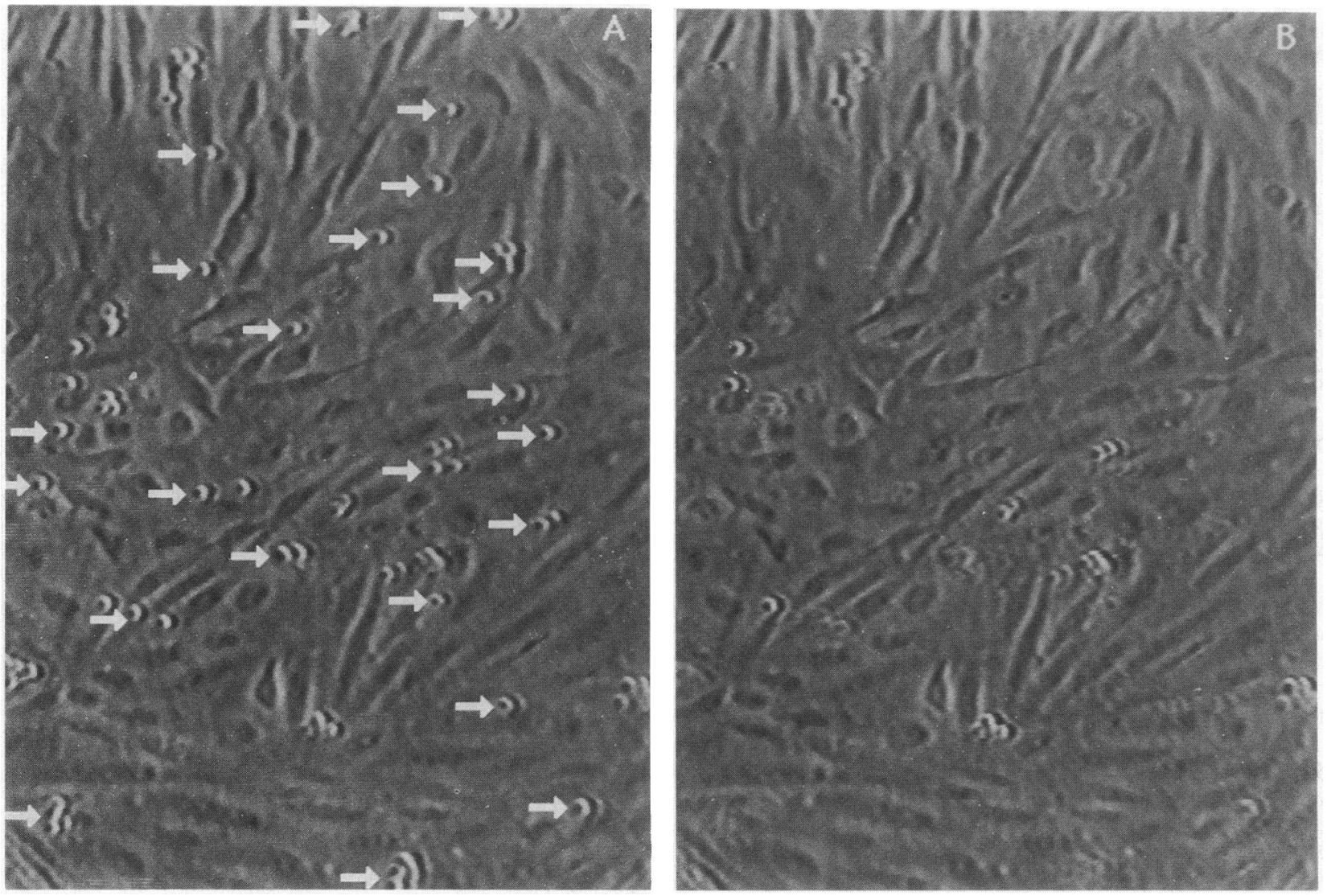

Figure 2. Digitized images of tumor cells firmly adherent and rolling on IL-1-activated HUVEC. The image in $(A)$ was digitized using singleframe acquisition, and both adherent and nonadherent cells are shown. The image in $(B)$ was digitized using the averaging technique (multiframe). Cells not firmly attached are indicated by arrows in $A$ but are not shown in $B$, which only includes firmly attached cells. Flow direction is indicated by direction of the arrows.

frame but not in the multiframe image. Rolling velocity of tumor cells was measured as the distance traveled by a cell in $5 \mathrm{~s}$. Rolling velocity in $\mu \mathrm{m} / \mathrm{s}$ was calculated from screen pixel coordinates using exact enlargement of the images measured by a reference grid and a micrometer eye-piece (Nachet Italia SRL, Milan, Italy). More than 30 cells in each experiment were used to calculate mean rolling velocity. Two images were compared by simultaneous display on the monitor screen. Measured parameters are expressed as mean $\pm \mathrm{SE}$.

\section{Results}

Adhesion of tumor cells on HUVEC. The adhesion behavior of human tumor cells to HUVEC under flow conditions is summarized in Table I. The number of rolling and adhering cells per unit surface area were measured after 12 min of perfusion, as described in Fig. 1. Two adhesion patterns were observed, depending on the different tumor types. The colon carcinoma (HT29M), the ovarian carcinoma (OVCAR-3), and the breast carcinoma (T-47D) cells showed little interaction with resting HUVEC, whereas they rolled and then attached on IL-1-activated HUVEC. In contrast, the melanoma A375M and A2058 and the osteosarcoma MG-63 cells already showed some adhesion on resting HUVEC and massive adhesion on IL-1-activated HUVEC, but no cell rolling was observed throughout the perfusion.

The kinetics of cell rolling and adhesion to HUVEC during perfusions, at different shear stress levels, was investigated in

Table I. Effect of IL-1 on Rolling and Adhesion of Human Tumor Cell Lines on HUVEC under Flow Conditions

\begin{tabular}{llllll}
\hline Tumor line & \multicolumn{1}{c}{ Origin } & \multicolumn{2}{c}{ Resting HUVEC } & IL-1-activated HUVEC \\
\hline HT-29M & Colon carcinoma & Low rolling (1) & Low adhesion (7) & Rolling (124) & Adhesion (566) \\
OVCAR-3 & Ovarian carcinoma & No rolling (0) & Low adhesion (4) & Rolling (27) & Adhesion (185) \\
T-47D & Breast carcinoma & Low rolling (3) & Low adhesion (18) & Rolling (15) & Adhesion (241) \\
A375M & Melanoma & No rolling (0) & Adhesion (102) & No rolling (0) & Adhesion (475) \\
A2058 & Melanoma & No rolling (0) & Adhesion (198) & No rolling (0) & Adhesion (712) \\
MG-63 & Osteosarcoma & No rolling (0) & Adhesion (65) & No rolling (0) & Adhesion (1136)
\end{tabular}

Tumor cell rolling and adhesion on HUVEC were evaluated after 12 min perfusion as shown in Fig. 1 . Number in parentheses are the mean number of cells per square millimeter, measured in two to six experiments. 


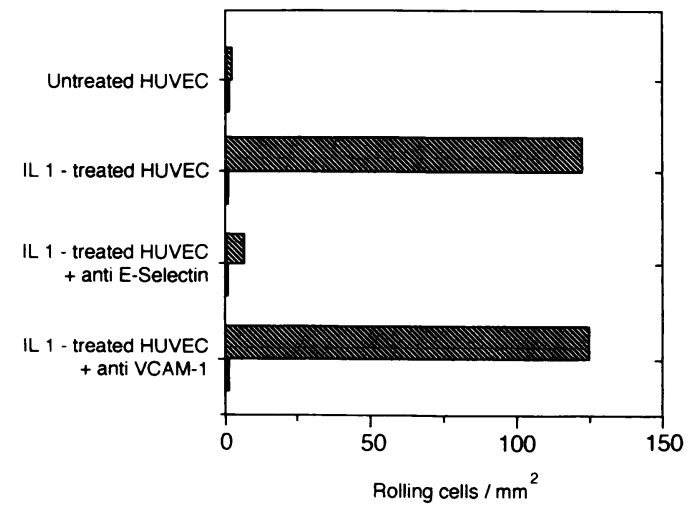

Figure 3. Rolling of HT-29M and A375M tumor cells on untreated and IL-1-treated HUVEC. Mean numbers of cells rolling and attached on the EC surface were evaluated at $3.0 \mathrm{dyn} / \mathrm{cm}^{2}$. Values are

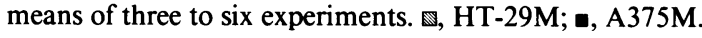

detail using the HT-29M colon carcinoma and the A375M melanoma. HT-29M colon carcinoma adhesion to HUVEC under flow conditions is illustrated in Figs. 3-5. Few tumor cells interacted with unstimulated HUVEC and some cells rolled on HUVEC (Fig. 3). On average, only $7 \pm 5$ cells $/ \mathrm{mm}^{2}$ adhered firmly to the endothelium after $12 \mathrm{~min}$ of perfusion (Fig. 5). On HUVEC stimulated with IL-1, a large number of HT-29M cells rolled and firmly adhered to the endothelium, as documented in the four images shown in Fig. 4. More than 120 cells $/ \mathrm{mm}^{2}$ on average were rolling on the HUVEC surface at
$3.0 \mathrm{dyn} / \mathrm{cm}^{2}$ (Fig. 3). The mean velocity of rolling HT-29M cells on IL-1-activated HUVEC averaged $6.4 \pm 3.4 \mu \mathrm{m} / \mathrm{s}$. In addition to rolling, HT-29M cells also firmly adhered to IL-1-stimulated HUVEC within a few seconds from the beginning of perfusion and the number of adherent cells rose constantly with decreasing shear stress. As shown in Fig. 5, when flow rate was stepped down (at 3,6, and 9 min perfusion time) the number of adherent cells concomitantly rose, suggesting that shear stress changes promote adhesion. By the end of the perfusion, a large number of adherent cells was observed ( $566 \pm 67$ cells $/ \mathrm{mm}^{2}$ on average, [Fig. 5]).

To study whether rolling and adhesion of HT-29M cells to IL-1-activated endothelium were mediated by E-selectin or by VCAM-1, we performed adhesion experiments after incubating IL-1-activated HUVEC with $\mathrm{mAb}$ against these two proteins. Treatment of stimulated HUVEC with $\mathrm{mAb}$ against Eselectin completely prevented rolling and adhesion of HT-29M cells (Figs. 3 and 5) at all shear stress levels and very few cells came into contact with the endothelium during perfusion. When IL-1-stimulated HUVEC were treated with mAb against VCAM-1, the number of HT-29M cells rolling and adhering were comparable to the counts for IL-1-activated HUVEC alone (Figs. 3 and 5).

The results of adhesion experiments with A375M melanoma cells are reported in Figs. 3 and 6. There was virtually no adhesion on resting HUVEC monolayers at high shear stress $\left(>1.5 \mathrm{dyn} / \mathrm{cm}^{2}\right)$, but cells began to adhere to unstimulated HUVEC at $0.6 \mathrm{dyn} / \mathrm{cm}^{2}$ (Fig. 6). After $12 \mathrm{~min}$ of perfusion the mean number of firmly attached cells reached $102 \pm 49$ cells/
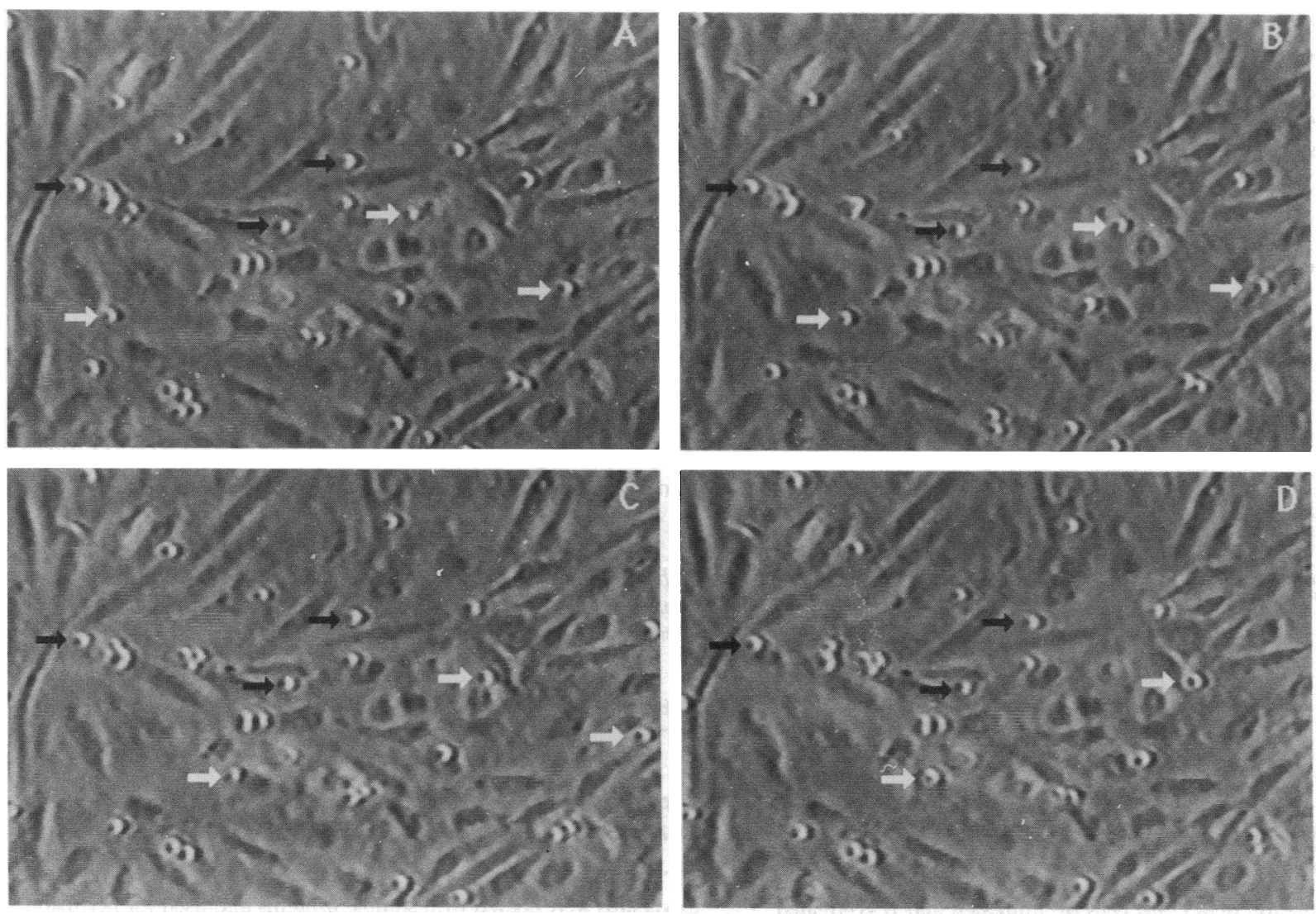

Figure 4. Digitized images of HT-29M cells adhering and rolling on IL-1-treated HUVEC. The four panels were digitized sequentially (3-s intervals between consecutive images). Black arrows indicate HT-29M cells firmly attached to the endothelium monolayer. White arrows indicate HT-29M cells rolling over the endothelial surface. Arrows also indicate the direction of flow. 

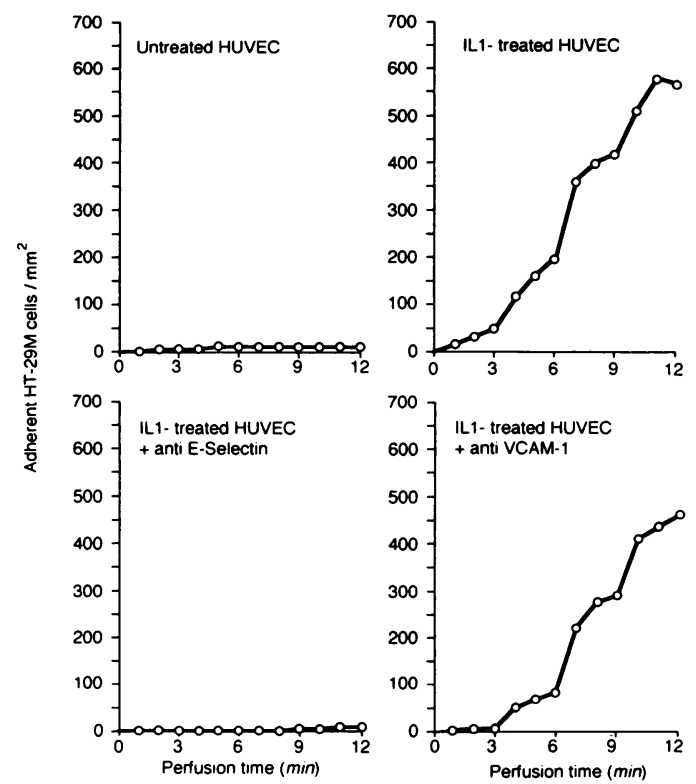

Figure 5. Adhesion of HT-29M cells on untreated and IL-1-treated HUVEC. Adhesion was measured at different wall shear stresses during perfusion (from 3.0 to $0.3 \mathrm{dyn} / \mathrm{cm}^{2}$ ), as reported in Fig. 1. Values are the mean of three to six experiments.

$\mathrm{mm}^{2}$. Unlike the HT-29M cells, A375M cells did not roll on the surface of HUVEC (Fig. 3). On HUVEC stimulated with IL-1, melanoma cells started to adhere even at the highest shear stress and, by the end of perfusion, a large number was adhering (on average $475 \pm 65$ cells $/ \mathrm{mm}^{2}$, Fig. 6 ). During the whole experiment, A375M cells showed only firm adhesion to the HUVEC, with no rolling on the cell surface (Fig. 3).

The effect of mAb against E-selectin and VCAM-1 on adhe-
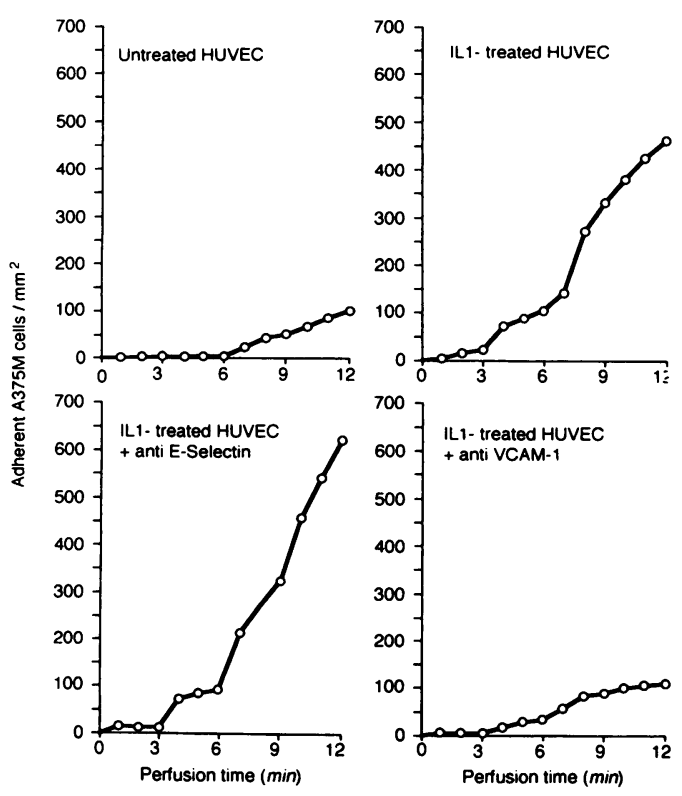

Figure 6. Adhesion of A375M cells on untreated and IL-1-treated HUVEC. Adhesion was measured at different wall shear stresses during perfusion ( from 3.0 to $0.3 \mathrm{dyn} / \mathrm{cm}^{2}$ ), as reported in Fig. 1. Values are the mean of three to six experiments. sion of A375M cells to IL-1-activated HUVEC were also studied. When the activated HUVEC were incubated with $\mathrm{mAb}$ against VCAM-1, adhesion was significantly prevented and, by the end of the perfusion, an average of only $103 \pm 59$ cells $/ \mathrm{mm}^{2}$ adhered (Fig. 6). On the contrary, mAb against E-selectin had no effect on adhesion of A375M cells (Fig. 6).

Adhesion of tumor cells on soluble proteins. To investigate in more detail how E-selectin and VCAM-1 mediate the interaction of flowing tumor cells with the vascular wall, we studied the adhesion of HT-29M and A375M cells on plastic surfaces coated with these adhesion proteins. These perfusions followed the same protocol as for HUVEC (Fig. 1). Rolling and adherent tumor cells were measured between 12 and $15 \mathrm{~min}$ of perfusion time at a wall shear stress of $3.0 \mathrm{dyn} / \mathrm{cm}^{2}$ ( Table II). No cell adhesion to uncoated surfaces due to unspecific cell binding was observed for either tumor cell line.

A large number of HT-29M cells rolled on the E-selectin coated surfaces, as observed on activated endothelial surface, though, unlike on HUVEC, almost all cells in contact with the surface rolled continuously throughout perfusion without firm attachment (Table II). Mean velocity of rolling cells on E-selectin averaged $5.4 \pm 2.0 \mu \mathrm{m} / \mathrm{s}$, comparable to the HT-29M cells on activated HUVEC. HT-29M cells, on the other hand, did not adhere or roll on the VCAM-1-coated surface, at any wall shear stress (Table II).

A375M melanoma cells neither rolled nor adhered on the E-selectin coated surface but they adhered largely on VCAM1-coated surfaces ( Table II). As shown for HUVEC, all cells in contact with the VCAM-1-coated surface were firmly attached, with no rolling. Rolling of HT-29M cells on immobilized E-selectin and adhesion of A375M cells on immobilized VCAM-1 were both blocked by incubating the protein-coated surfaces with the respective mAb (data not shown).

\section{Discussion}

The interaction of blood-borne tumor cells with vascular endothelium is a key component of hematogenous spread. To adhere to the vessel wall, tumor cells that come into contact with the microvasculature must resist the tractive forces of the flow of plasma and circulating cells that tend to detach them from the wall (34). Studies of the mechanisms involved in the processes of adhesion must, therefore, take account of the physical forces acting on individual cells.

We investigated the adhesive properties of different human tumor cells under physiological flow conditions such as those

Table II. Number of Rolling and Adherent Tumor Cells on Protein-coated Surfaces

\begin{tabular}{lccccc}
\hline & \multicolumn{2}{c}{ HT-29M } & & \multicolumn{2}{c}{ A375M } \\
\cline { 2 - 3 } \cline { 5 - 6 } Substrate & Rolling & Adherent & & Rolling & Adherent \\
\hline \multicolumn{2}{c}{ cells $/ \mathrm{mm}^{2}$} \\
E-selectin & $316(281-352)$ & $2(1-3)$ & & & cells $/ \mathrm{mm}^{2}$ \\
VCAM-1 & 0 & $1(0-3)$ & & 0 & $1(0-3)$ \\
& & & & &
\end{tabular}

Coverslips were coated with soluble proteins and used for perfusion experiments as described in Fig. 1. Tumor cell adhesion was evaluated after $12 \mathrm{~min}$ perfusion. Cell rolling was evaluated at $3.0 \mathrm{dyn} / \mathrm{cm}^{2}$. Results represent the mean (range) of three to four experiments. 
in postcapillary venules $(11,35,36)$. Only few colon carcinoma (HT-29M), ovarian carcinoma (OVCAR-3), and breast carcinoma (T-47D) cells adhered to resting HUVEC at the shear stress values tested during the perfusion experiments (0.3-3.0 dyn $/ \mathrm{cm}^{2}$ ). However, melanoma (A375M and A2058) and osteosarcoma (MG63) cells adhered to nonactivated HUVEC at low shear stresses $\left(<0.6 \mathrm{dyn} / \mathrm{cm}^{2}\right.$ for A375M melanoma). This suggests these tumor cells have adhesion structures on their surface that promote the interaction with resting EC. Glycosphyngolipid-glycosphyngolipid has been recently suggested as the main mediator of the initial adhesion of murine melanoma variants to nonactivated EC under flow conditions (37).

As previously observed under static conditions, cytokine activation of HUVEC increased tumor cell adhesion (16-18). This increase is mediated by the induction, or enhanced expression, of adhesion molecules on EC (19). All the tumor cell lines investigated under flow conditions adhered to IL-1-activated HUVEC already at the highest shear stress level tested, and adhesion increased as shear stress decreased. However, two different mechanisms characterized the adhesion of tumor cells to activated HUVEC. Colon, breast, and ovarian carcinoma cells adhered on EC surface with rolling, whereas melanoma and osteosarcoma cells adhered to activated HUVEC without rolling. Leukocyte rolling on the EC surface has been well documented in vivo and in vitro $(4,6,9,11)$. Rolling velocity in vivo ranges from 10 to $20 \mu \mathrm{m} / \mathrm{s}$ and depends linearly on shear stress (5). Our estimated mean rolling velocity for HT-29M cells $\left(\sim 6 \mu \mathrm{m} / \mathrm{s}\right.$ at $\left.3.0 \mathrm{dyn} / \mathrm{cm}^{2}\right)$ corresponds well to the values measured for leukocytes. It would thus appear that for some tumor cell types, rolling on the EC surface is a prerequisite for subsequent firm adhesion, as for polymorphonuclear leukocytes (12). At the same time, other tumor cell types seem to use different mechanisms of interaction to adhere to the EC in the presence of flow, without requiring rolling.

In line with our observation, it has been reported that different tumor types use different adhesion pathways. A specific role has been proposed for E-selectin in the adhesion of colon carcinomas, including HT-29M, to activated EC $(20,21)$, whereas VCAM-1 appears preferentially to mediate the adhesion of melanomas $(20,22,24)$. We used mAb against these two adhesion proteins to clarify the mechanisms of H'T-29M and A375M cell adhesion under flow conditions. E-selectin played a major role in mediating rolling and subsequent adhesion of HT-29M colon carcinoma cells, since its inhibition by a $\mathrm{mAb}$ anti E-selectin completely prevented both processes, but anti E-selectin had no effect on the adhesion of A375M melanoma cells that adhered on EC without rolling. These results extend previous observations on the specific role of selectins in the rolling phase of cell adhesion (12). In contrast, VCAM-1 is certainly involved in adhesion of A375M melanoma cells, since its inhibition by mAb against VCAM-1 prevented their firm adhesion to activated EC, thus suggesting it has a specific role in the stable binding of circulating cells to the EC surface.

That these two classes of adhesive proteins specifically mediate two different aspects of tumor cell adhesion is further confirmed by our observations on tumor cell interactions with immobilized soluble proteins under flow conditions. HT-29M colon carcinoma cells rolled on E-selectin-coated surfaces but not on surfaces coated with VCAM-1. A375M melanoma cells adhered on VCAM-1 without rolling, but neither adhered nor rolled on E-selectin coated surfaces. Thus, under flow condi- tions, E-selectin has a selective role in cell rolling and VCAM-1 in firm adhesion. All the tumor cell types that rolled on activated HUVEC under flow (Table I) preferentially adhered on the E-selectin-coated surface in static conditions, whereas tumor cells that firmly attached on HUVEC without rolling in flow preferentially adhered on VCAM-1-coated surface in static conditions (Giavazzi, R., unpublished observation). The adhesion of HT-29M cells to the E-selectin-coated surface under static conditions contrasts somewhat with the lack of adhesion under flow conditions ( Table II). Our interpretation is that HT-29M cells bind weakly but continuously to E-selectin, so that when the forces induced by motion of the fluid act on the cells, they continuously move on the surface without detachment. This implies that adhesion of tumor cells to immobilized protein is quantifiable under static conditions, whereas when they are subjected to flow, they continuously roll without firm adhesion.

In conclusion, our results indicate that the interaction of human tumor cells with EC under flow conditions involves rolling and firm adhesion, depending on the respective adhesive properties of the endothelial and tumor cells. Thus, some tumor cell types roll and subsequently adhere on the endothelial surface using selectin-mediated molecules (i.e., E-selectin), while others adhere without rolling using integrin-mediated receptors on EC (i.e., VCAM-1). Since specific adhesion mechanisms play a major role in tumor cell interactions with the vascular endothelium, it would be useful to characterize these processes to shed more light on the metastatic potential of tumor cells and the organ preference of metastasis.

\section{Acknowledgments}

We thank Dr. Marina Figliuzzi and Mr. Renato Chirivi for assistance in cell culture, and Mrs. Judy Baggott for editing the manuscript. We also wish to thank Dr. E. Dejana for critically reviewing the manuscript.

This work was supported in part by a research grant from the Italian Association for Cancer Research.

\section{References}

1. Nicolson, G. L. 1988. Organ specificity of tumor metastasis: role of preferential adhesion, invasion and growth of malignant cells at specific secondary sites. Cancer Metastasis Rev. 7:143-188.

2. Giavazzi, R., and G. Taraboletti. 1991. Endothelium-tumor cell interaction in the metastatic process. In Vascular Endothelium: Interactions with Circulating Cells. J. L. Gordon, editor. Elsevier Science Publishers B. V., Amsterdam. pp. 199-212.

3. Belloni, P. N., and R. J. Tressler. 1989. Microvascular endothelial cell heterogeneity: interactions with leukocytes and tumor cells. Cancer Metastasis Rev. 8:353-389.

4. Zimmerman, G. A., S. M. Prescott, and T. M. McIntyre. 1992. Endothelial cell interactions with granulocytes: tethering and signaling molecules. Immunol. Today 13:93-100.

5. Atherton, A., and G. V. R. Born. 1973. Relationship between the velocity of rolling granulocytes and that of the blood flow in venules. J. Physiol. 233:157165.

6. Lay, K., P. Gaehtgens, C. Fennie, M. S. Singer, L. A. Lasky, and S. D. Rosen. 1991. Lectin-like cell adhesion molecule 1 mediates leukocyte rolling in mesenteric venules in vivo. Blood. 77:2553-2555.

7. Lasky, L. A. 1992. Selectins: interpreters of cell-specific carbohydrate information during inflammation. Science (Wash. DC) 258:964-969.

8. Butcher, E. C. 1991. Leukocyte-endothelial cell recognition: three (or more) steps to specificity and diversity. Cell. 67:1033-1036.

9. Hogg, N. 1992. Roll, roll, roll your leucocyte gently down the vein. . . . Immunol. Today. 13:113-115.

10. Lawrence, M. B., L. V. McIntire, and S. G. Eskin. 1987. Effect of flow on polymorphonuclear leukocyte/endothelial cell adhesion. Blood. 70:1284-1290.

11. Lawrence, M. B., C. W. Smith, S. G. Eskin, and L. V. McIntire. 1990. Effect on venous shear stress on CD18-mediated neutrophil adhesion to cultured endothelium. Blood. 75:227-237. 
12. Lawrence, M. B., and T. A. Springer. 1991. Leukocytes roll on a selectin at physiologic flow rates: distinction from and prerequisite for adhesion through integrins. Cell. 65:859-873.

13. Smith, C. W., T. K. Kishimoto, O. Abbas, B. Hughes, R. Rothlein, L. V. McIntire, E. Butcher, and D. C. Anderson. 1991. Chemotactic factors regulate lectin adhesion molecule-1-dependent neutrophil adhesion to cytokine-stimulated endothelial cells in vitro. J. Clin. Invest. 87:609-618

14. Raz, A., and R. Lotan. 1987. Endogenous galactoside-binding lectins: a new class of functional tumor cell surface molecules related to metastasis. Cancer Metastasis Rev. 6:433-452.

15. Honn, K. V., and D. G. Tang 1992. Adhesion molecules and tumor cell interaction with endothelium and subendothelial matrix. Cancer Metastasis Rev. 11:353-375.

16. Dejana, E., F. Bertocchi, M. C. Bortolami, A. Regonesi, A. Tonta, F. Breviario, and R. Giavazzi. 1988. Interleukin 1 promotes tumor cell adhesion to cultured human endothelial cells. J. Clin. Invest. 82:1466-1470.

17. Rice, G. E., M. A. Gimbrone, and M. P. Bevilacqua. 1988. Tumor cell-endothelial interactions. Increased adhesion of human melanoma cells to activated vascular endothelium. Am. J. Pathol. 133:204-210.

18. Lauri, D., M. C. Bertomeu, F. W. Orr, E. Bastida, D. Sauder, and M. R. Buchanan. 1990. Interleukin-1 increases tumor cell adhesion to endothelial cells through an RGD dependent mechanism: in vitro and in vivo studies. Clin. Exp. Metastasis. 8:27-32.

19. Osborn, L. 1990. Leukocyte adhesion to endothelium in inflammation. Cell. 62:3-6.

20. Rice, G. E., and M. P. Bevilacqua. 1989. An inducible endothelial cell surface glycoprotein mediates melanoma adhesion. Science (Wash. DC). 246:1303-1306

21. Lauri, D., L. Needham, I. Martin-Padura, and E. Dejana. 1991. Tumor cell adhesion to endothelial cells: ELAM-1 as an inducible adhesive receptor specific for colon carcinoma cells. J. Natl. Cancer Inst. 83:1321.

22. Dejana, E., I. Martin-Padura, D. Lauri, S. Bernasconi, M. R. Bani, A. Garofalo, R. Giavazzi, J. Magnani, A. Mantovani, and S. Menard. 1992. Endothelial leukocyte adhesion molecule-1 dependent adhesion of colon carcinoma cells to vascular endothelium is inhibited by an antibody to Lewis fucosylated type I carbohydrate chain. Lab. Invest. 66:324-330.

23. Takada, A., K. Ohmori, T. Yoneda, K. Tsuyuoka, A. Hasegawa, K. Makoto, and R. Kannagi. 1993. Contribution of carbohydrate antigens sialyl Lewis A and sialyl Lewis $\mathrm{X}$ to adhesion of human cancer cells to vascular endothelium. Cancer Res. 53:354-361.

24. Martin-Padura, I., R. Mortarini, D. Lauri, S. Bernasconi, F. Sanchez-Madrid, G. Parmiani, A. Mantovani, A. Anichini, and E. Dejana. 1991. Heterogeneity in human melanoma cell adhesion to cytokine activated endothelial cells correlate with VLA-4 expression. Cancer Res. 51:2239-2241.
25. Lauri, D., I. Martin-Padura, T. Biondelli, G. Rossi, S. Bernasconi, R. Giavazzi, F. Passerini, V. Van Hinsbergh, and E. Dejana. 1991. Role of $\beta_{1}$ integrins in tumor cell adhesion to cultured human endothelial cells. Lab. Invest. 65:525-531.

26. Price, J. E., L. M. Daniels, D. E. Campbell, and R. Giavazzi. 1989. Organ distribution of experimental metastases of a human colorectal carcinoma injected in nude mice. Clin. Exp. Metastasis. 7:55-89.

27. Hamilton, T. C., R. C. Young, W. M. McKoy, K. R. Grotzinger, J. A. Green, E. W. Chu, J. Whang-Peng, A. M. Rogan, W. R. Green, and R. F. Ozols. 1983. Characterization of a human ovarian carcinoma cell line (NIH:OVCAR3) with androgen and estrogen receptors. Cancer Res. 43:5379-5389.

28. Engel, L. W., and N. A. Young. 1978. Human breast carcinoma cells in continuous culture: a review. Cancer Res. 38:4327-4336.

29. Kozlowski, J. M., I. R. Hart, I. J. Fidler, and N. Hanna. 1984. A human melanoma line heterogeneous with respect to metastatic capacity in athymic nude mice. J. Natl. Cancer Inst. (Bethesda). 72:913-917.

30. Todaro, G. J., C. Fryling, and J. E. De Larco. 1980. Transforming growth factors produced by certain human tumor cells: polypeptides that interact with epidermal growth factor receptor. Proc. Natl. Acad. Sci. USA. 77:5258-5262.

31. Bevilacqua, M. P., J. S. Pober, M. E. Wheeler, R. S. Cotran, and M. A. Gimbrone, Jr. 1985. Interleukin 1 acts on cultured human vascular endothelium to increase the adhesion of polymorphonuclear leukocytes, monocytes, and related leukocyte cell lines. J. Clin. Invest. 76:2003-2011.

32. Simmons, D. L., and L. Needham. 1991. Cloning cell surface molecules using monoclonal antibodies. In Vascular Endothelium: Interactions with Circulating Cells. J. L. Gordon, editor. Elsevier, Science Publishers B. V., Amsterdam. pp. 3-29.

33. Kotovuori, P., E. Tontti, R. Pigott, M. Shepherd, M. Kiso, A. Hasegawa, R. Renkonen, P. Nortamo, D. C. Altieri, and C. G. Gahmberg. 1993. The vascular E-selectin binds to the leukocyte integrins CD11/CD18. Glycobiology. 3:131136.

34. Weiss, L. 1992. Biomechanical interactions of cancer cells with the microvasculature during hematogenous metastasis. Cancer Metastasis Rev. 11:227235.

35. Schmid-Schoenbein, G. W., Y. C. Fung, and B. W. Zweifach. 1975. Vascular endothelium leukocyte interactions: sticking shear force in venules. Circ. Res. 36:173-184.

36. Schmid-Schoenbein, G. W., S. Usami, R. Skalak, and S. Chien. 1980. The interactions of leukocytes and erythrocytes in capillary and post-capillary vessels. Microvasc. Res. 19:45-70.

37. Kojima, N., M. Shiota, Y. Sadhira, K. Handa, and S.-I. Hakomori. 1992 Cell adhesion in a dynamic flow system as compared to static system. J. Biol. Chem. 267:17264-17270. 